\title{
Commentary A multigene RT-PCR assay used to predict recurrence in early
breast cancer: two presentations with contradictory results
} Willem E Fiets and Johan WR Nortier

\author{
Department of Medical Oncology, Leiden University Medical Center, The Netherlands \\ Corresponding author: Willem E Fiets, W.E.Fiets@lumc.nl
}

Published: 28 June 2004

Breast Cancer Res 2004, 6:185-187 (DOI 10.1186/bcr911)

(C) 2004 BioMed Central Ltd

\begin{abstract}
During the 2003 San Antonio Breast Cancer Symposium two studies were presented that were designed to validate a recurrence score, derived from a 21-gene RT-PCR assay, in patients with axillary node-negative breast cancer. This recurrence score was highly predictive for the risk of recurrence in 668 patients treated in a large multicenter trial with adjuvant tamoxifen. However, no prognostic value was found in a small group of patients who were retrospectively selected in a single institution and who did not receive any adjuvant systemic therapy. Further validation is needed to establish the prognostic and predictive role of this assay in clinical management.
\end{abstract}

Keywords breast cancer, predictive marker, prognostic marker, RNA

During the San Antonio Breast Cancer Symposium in December 2003 two studies were presented that were designed to validate a recurrence score, derived from a multigene RT-PCR assay, in patients with axillary nodenegative (ANN) breast cancer $[1,2]$. Contradictory results were reported from these studies. The multigene RT-PCR recurrence score accurately predicted the likelihood of distant metastasis in a cohort of 668 patients with ANN breast cancer treated with tamoxifen [1], but failed to predict recurrence risk in 149 patients who had not received any adjuvant systemic therapy [2].

The need for identifying new prognostic markers in ANN breast cancer, such as the multigene RT-PCR recurrence score, is highly relevant. Risk categories for recurrence are currently defined according to consensus-based guidelines using histological and clinical characteristics [3]. Following these guidelines, up to $85 \%$ of ANN breast cancer patients are candidates for adjuvant systemic treatment. However, as many as $70-80 \%$ of these patients do not develop distant metastases, and therefore do not need adjuvant treatment [4]. These patients are unnecessarily exposed to short-term and long-term side effects. In order to define risk categories more precisely, a large number of biologic and molecular markers have been investigated. However, to date, these often promising markers cannot be incorporated into clinical practice due to the lack of standard validation, poor reproducibility, or weak prognostic power [5].

Recent advances in our knowledge of the human genome have led to the development of powerful high-performance screening techniques for molecular analysis. A 70-gene prognosis profile determined by microarray analysis appeared strongly predictive for outcome in young patients with breast cancer [6,7], and is presently in the process of validation in other series of patients in different laboratories. A major obstacle for the broad application of microarray analysis will be the requirement of fresh tumor tissue. This is necessary as RNA degrades into small fragments in formalin-fixed paraffin-embedded tissue. RTPCR is especially suitable for the analysis of small fragments of RNA and can accurately measure individual genes of interest in formalin-fixed paraffin-embedded tissue [8]. A 92-gene RT-PCR assay on archival formalinfixed paraffin-embedded breast cancer tissue has been 
shown to be sensitive, specific, and highly reproducible [9]. Measured levels of estrogen receptor (ER) mRNA, progesterone receptor mRNA and HER2 mRNA were concordant with the levels of the respective proteins measured by immunohistochemistry [9].

The recurrence score presented at the San Antonio Breast Cancer Symposium by Paik and colleagues [1] and by Esteva and colleagues [2] was derived from a 21-gene RT-PCR assay containing 16 cancer-related genes and five reference genes. Three studies, including a total of 447 patients, were used to select the 21 genes from a panel of 250 candidate genes with known prognostic value $[1,10,11]$. The 16 cancer-related genes were divided into seven clusters. Higher expression of HER2 (HER2, GRB7), of proliferation markers (Ki-67, STK15, survivin, cyclin B1, MYBL2), of invasion markers (stromolysin 3, cathepsin L2) and of the macrophage marker CD68 was associated with shorter relapse-free survival. Higher expression of estrogen-associated genes (ER, progesterone receptor, Bcl2, SCUBE2), GSTM1 and $B A G 1$ were associated with longer relapse-free survival.

An algorithm was developed to quantify the recurrence score. Most accent was given to the proliferation-related genes (+1.04), to the HER2 genes $(+0.47)$, and to the estrogen-associated genes (-0.34). The maximum achievable recurrence score was 100. A recurrence score less than 18 was considered low risk of recurrence, a score greater than or equal to 31 was considered high risk of recurrence, and the remaining scores were considered intermediate risk of recurrence.

Paik and colleagues presented a validation study of the 21-gene RT-PCR recurrence score that evaluated 668 of the 2617 patients enrolled in the tamoxifen arm of the NSABP B-14 trial. This arm of the trial randomized postmenopausal patients with ER-positive ANN breast cancer to placebo or to tamoxifen. The distribution of the various risk categories was $51 \%$ low risk, 22\% intermediate risk, and $27 \%$ high risk. The overall 10 -year distant relapse-free survival (DRFS) rate was 85\%. For patients with a low recurrence score, the 10-year DRFS rate was 93.2\%; for patients with an intermediate recurrence score, the DRFS rate was $85.7 \%$; and for patients with a high recurrence score, the DRFS rate was $69.5 \%$.

These data confirm that this multigene RT-PCR-derived recurrence score could predict relapse-free survival fairly accurately in ANN breast cancer patients treated with tamoxifen $(P<0.00001)$. Multivariate analysis showed that it did so independently of age and tumor size. As this recurrence score is a continuous variable, a prediction for recurrence, with a $95 \%$ confidence interval, can be made
Somewhat to the contrary, Esteva and colleagues were unable to confirm these results in a group of patients who did not receive any form of adjuvant therapy. They intended to validate the recurrence score in a selection of patients taken from a cohort of 4428 patients with ANN breast cancer who received primary surgical therapy, and radiotherapy if indicated, at the Texas MD Anderson Cancer Center between 1978 and 1995. Identified were 220 patients who received no adjuvant systemic therapy and whose tumor blocks were available and contained at least 5\% invasive cancer. Tumor blocks from 149 patients were evaluable. Sixty-nine percent of these patients were ER-positive. Thirty-two percent of patients fell into the lowrisk group, $14 \%$ into the intermediate-risk group, and 54\% into the high-risk group. For patients with a low recurrence score, the 10-year DRFS rate was $82 \%$; for patients with an intermediate recurrence score, the DRFS rate was $62 \%$; and for patients with a high recurrence score, the DRFS rate was $78 \%$. No significant correlation between the 21 gene RT-PCR recurrence score and DRFS was found.

Analysis of standard risk factors revealed an unexpected association between higher nuclear grade and lower risk of recurrence $(P=0.02)$. This association could have negatively influenced the prognostic value of the recurrence score in this cohort, as proliferation markers are dominant denominators in the algorithm that determines the recurrence score. Another potential explanation for the discrepant findings of this small group of patients is selection bias. No more than 5\% of ANN breast cancer patients treated at the Texas MD Anderson Cancer Center during a 17-year period were identified to be included in this study. Of these patients, only $68 \%$ were evaluable for analysis. On the contrary, $99 \%$ of eligible patients in the study presented by Paik and colleagues were evaluable for analysis.

It might also be possible that the recurrence score is useful in predicting response or lack of response to tamoxifen of ER-positive patients, but is not a good predictor for recurrence in general. The recurrence score was developed in three groups of patients who were almost all treated with tamoxifen and/or chemotherapy. Some of the markers in the panel may be more predictive than prognostic. The estrogen-related markers are considered to be weak prognostic factors after longer follow-up, but to be strong predictors for the response to hormonal therapy [5]. HER2 overexpression appears to be only a weak unfavorable prognostic factor [5], but is highly predictive for treatment with trastuzumab and shows a negative association with the benefit of tamoxifen $[12,13]$.

Using the St Gallen criteria, approximately $15 \%$ of all ANN patients are classified as low risk (grade I, $<2 \mathrm{~cm}$, and ERpositive). The recommendations for these patients are to treat them either with adjuvant endocrine therapy or with 
no adjuvant therapy. For all other ANN patients with ERpositive tumors, the St Gallen consensus considers both endocrine therapy alone and the combination of chemotherapy and endocrine therapy treatment options. The threshold above which combination chemotherapy and endocrine therapy is advised is not well defined, but should include consideration of the degree of hormonereceptor positivity, the presence of HER2 overexpression, the tumor grade, and the proliferation rate [3].

In particular, the genes that dominate the algorithm determined from the 21-gene RT-PCR-derived recurrence score represent these features. The recurrence score may thus be a better tool to discriminate between ANN ERpositive patients who can be treated with endocrine therapy alone (low risk), and those who may benefit from the combination of endocrine therapy and chemotherapy (intermediate risk and high risk). However, the discriminative effect of the recurrence score appears no better than some known prognostic factors or combinations of known prognostic factors, such as urokinase-type plasminogen activator and plasminogen activator inhibitor-1 [14]. In addition, it is not clear whether intermediate-risk and high-risk patients benefit from chemotherapy on top of endocrine therapy. Paik and colleagues analyzed tumors from the tamoxifen only arm of the NSABP-B20 trial for the 21-gene recurrence score [1]. It would have been helpful if tumors from the chemotherapy plus tamoxifen arm were studied as well.

In conclusion, gene profiles determined by multigene RTPCR analyses potentially provide powerful tools to tailor adjuvant systemic treatment. The 21-gene RT-PCR assay presented at the San Antonio Breast Cancer Symposium 2003 was highly predictive for the risk of recurrence in 668 ANN patients who participated in the NSABP-B14 study and who were treated with adjuvant tamoxifen. However, no prognostic value was found in a small highly selected group of patients with ANN breast cancer who did not receive any adjuvant systemic therapy. Further validation is needed, particularly for the untreated patients, and quality control has to be assured. More studies are needed to establish the prognostic and predictive role of these assays in clinical management.

\section{Competing interests}

None declared.

\section{References}

1. Paik S, Shak S, Tang G, Kim C, Baker J, Cronin M, Baehner R, Walker M, Watson D, Park T, et al:: Multi-gene RT-PCR assay for predicting recurrence in node negative breast cancer patients - NSABP studies B-20 and B-14 [abstract]. Breast Cancer Res Treat 2003, 82:A16 [http://www.sabcs.org].

2. Esteva FJ, Sahin AA, Coombes K, Baker J, Cronin M, Walker M, Watson D, Cristofanilli M, Shak S, Hortobagyi GN: Multi-gene RT-PCR assay for predicting recurrence in node negative breast cancer patients - M.D. Anderson Clinical Validation Study [abstract]. Breast Cancer Res Treat 2003, 82:A17 [http://www.sabcs.org].
3. Goldhirsch A, Wood WC, Gelber RD, Coates AS, Thürlimann B, Senn H-J: Meeting highlights: updated international expert consensus on the primary therapy of early breast cancer. J Clin Oncol 2003, 21:3357-3365.

4. Caldas C, Aparicio AJ: The molecular outlook. Nature 2002, 415:484-485.

5. Isaacs C, Strearns V, Hayes DF: New prognostic factors for breast cancer recurrence. Semin Oncol 2001, 28:53-67.

6. van 't Veer LJ, Dai H, van de Vijver MJ, He YD, Hart AM, Mao M, Peterse $\mathrm{HL}$, van der Kooy K, Marton MJ, Witteveen AT, et al:: Gene expression profiling predicts clinical outcome of breast cancer. Nature 2002, 415:530-536.

7. van de Vijver M, He YD, van 't Veer LJ, Dai H, Hart AAM, Voskuil DW, Schreiber GJ, Peterse JL, Roberts C, Marton MJ, et al.: A gene-expression signature as a predictor of survival in breast cancer. N Engl J Med 2002, 347:1999-2009.

8. Lewis F, Maughan NJ, Smith V, Hillan K, Quirke P: Unlocking the archive - gene expression in paraffin-embedded tissue. J Patho/ 2001, 195:66-71.

9. Cronin M, Pho M, Dutta D, Stephans JC, Shak S, Kiefer MC, Esteban JM, Baker JB: Measurement of gene expression in archival paraffin-embedded tissues. Development and performance of a 92-gene reverse transcriptase-polymerase chain reaction assay. Am J Pathol 2004, 164:35-42.

10. Cobleigh MA, Bitterman P, Baker J, Cronin M, Liu M-L, Borchik R, Tabesh B, Mosquera J-M, Walker MG, Shak S: Tumor gene expression predicts distant disease-free survival (DDFS) in breast cancer patients with 10 or more positive nodes: high throughput RT-PCR assay of paraffin-embedded tumor tissues [abstract]. Proc Am Soc Clin Oncol 2003, 22:A3415.

11. Esteban J, Baker J, Cronin M, Liu M-L, Llamas MG, Walker MG, Mena R, Shak S: Tumor gene expression and prognosis in breast cancer: multi-gene RT-PCR assay of paraffin-embedded tissue [abstract]. Proc Am Soc Clin Oncol 2003, 22: A3416.

12. Carlomagno $C$, Perrone $F$, Gallo $C$, de Laurentiis $M$, Lauria $R$, Morabito A, Pettinato G, Panico L, D'Antonio A, Bianco AR, et al.: c-erbB2 overexpression decreases the benefit of adjuvant tamoxifen in early-stage breast cancer without axillary lymph node metastases. J Clin Oncol 1996, 14:2702-2708.

13. Ellis $M J$, Coop $A$, Singh $B$, Mauriac $L$, Llombert-Cussac $A$, Jänicke F, Miller WR, Evans DB, Dugan M, Brady C, et al.: Letrozole is more effective neoadjuvant endocrine therapy than tamoxifen for ErbB-1- and/or ErbB-2-positive, estrogen receptor-positive primary breast cancer: evidence from a phase III randomized trial. J Clin Oncol 2001, 19:3808-3816.

14. Look MP, van Putten WLJ, Duffy MJ, Harbeck N, Christensen IJ, Thomssen C, Kates R, Spyratos F, Fernö M, Eppenberger-Castori $\mathrm{S}$, et al:: Pooled analysis of prognostic impact of urokinasetype plasminogen activator and its inhibitor PAl-1 in 8377 breast cancer patients. J Natl Cancer Inst 2002; 94:116-128. 